\title{
Black hole mass spectrum vs spectrum of Hawking radiation
}

\author{
V.A.Berezin \\ Institute for Nuclear Research of the Russian Academy of Sciences, 60th October Anniversary \\ Prospect, 7a, 117312, Moscow, Russia e-mail: berezin@ms2.inr.ac.ru \\ A.M.Boyarsky, A.Yu.Neronov \\ Dept. of Mathematics and Mechanics, Lomonosov Moscow State University, 119899, Moscow, \\ Russiae-mail: boyarsk@mech.math.msu.su,aneronov@mech.math.msu.su
}

(June 19, 2021)

\begin{abstract}
We consider a massive selfgravitating shell as a model for collapsing body and a null selfgravitating shell as a model for quanta of Hawking radiation. It is show that the mass-energy spectra for the body and the radiation do not match. The way out of this difficulty is to consider not only out-going radiation but also the ingoing one. It means that the structure of black hole is changing during its evaporation resulting in the Bekenstein-Mukhanov spectrum for large masses.
\end{abstract}

Pacs number(s): 04.70.Dy,04.20.Gz

Typeset using REVTEX 
It is well known that in classical physics any spherically symmetric neutral black hole state is described by the single parameter, the total mass (Schwarzschild mass, or total energy) of this black hole. And after the black hole formation all the information about the details of the collapse is lost. In particular, the black hole can be formed either due to the bound motion of matter or due to its unbound motion. In quantum mechanics we are used to the fact that we have a discrete mass (energy) spectrum for a bound motion and a continuous spectrum for an unbound motion. We have now two possibilities. Either the black hole total mass has a discrete spectrum or its spectrum is continuous. If it is continuous, then it is not clear, first, why in the case of bound motion (which is, of course, also possible) the spectrum is continuous and, second, how to construct a ground state with nonzero mass which, we have reasons to believe, exists. If it is discrete, then the corresponding discrete quantum number has the same origin both for bound and unbound motions. But the bound motion has also a "conventional" quantum number (or numbers). And the unbound motion has also a "conventional" continuous parameter (or parameters). From this it follows that if the mass of the black hole is quantized (e.i., discrete), the quantum black hole state is described not only by its mass but also by some other parameter(s), discrete or continuous.

But if this is the case, the spectrum of radiation coming from a black hole should have a very complicated structure. Indeed, according to the Bohr postulate, the spectrum of emitted quanta must correspond to the energy level spacing of quantum system. If the black hole spectrum depends on extra parameters, the radiation spectrum would have a fine structure in the case of discrete additional parameters or it is continuous in the case of continuous additional parameters. So we have to conclude that the radiation spectrum "remembers" the way how the black hole was formed. The spectrum seems to be not the universal one. But in accordance with the well known semiclassical result by S.Hawking, the spectrum of radiation from a black hole is universal - it is thermal with the temperature $T_{B H}=m_{p l}^{2} / 8 \pi m$ where $m$ is the mass of the Schwarzschild black hole.

We are led to a contradiction. If we consider radiation propagating on the black hole background we have to conclude that it has the same spectrum no matter how the black hole 
was formed. This property mimics the classical property that the only parameter describing a black hole is its mass. On the other side if we calculate the spectrum of radiation from the black hole mass spectrum, we see that it depends on how the black hole was formed and is different for different black holes with equal masses.

In this paper we demonstrate on a simple quantum black hole model (see [2] for the detailed description) how this contradiction could be resolved. The black hole spectrum has a complicated structure with all the properties discussed above. Nevertheless, the spectrum of radiation is universal for the black holes with Schwarzschild mass $m$.

The main effect which enables us to resolve the contradiction is the following. We take fully into account the effect of back reaction of radiation on the gravitational field of the black hole. We show that only radiation with particular (discrete) spectrum could propagate from the black hole self-consistently. This turns out to be the property which defines the universal spectrum of Hawking radiation from black hole.

We consider the following quantum black hole model. The black hole is formed by a spherically symmetric collapsing thin shell (Fig. 1). There are different types of space-times formed either by bound (a) or unbound (b) motion of the shell. and The corresponding Hamiltonian formalism has been extensively studied [[],2] The result is that we have the only nontrivial equation [2]

$$
\begin{gathered}
e^{G P_{R} / R}=\frac{1}{2 \sqrt{F_{\text {in }} F_{\text {out }}}}\left(F_{\text {in }}+F_{\text {out }}-\frac{M^{2} G^{2}}{R^{2}} \pm \frac{2 M G}{R} \mathcal{Z}\right) \\
\mathcal{Z}=\sqrt{\left(F_{\text {out }}-F_{\text {in }}\right)^{2} \frac{R^{2}}{4 M^{2} G^{2}}-\frac{1}{2}\left(F_{\text {out }}+F_{\text {in }}\right)+\frac{M^{2} G^{2}}{4 R^{2}}}
\end{gathered}
$$

where $M$ is bare mass of the shell, $R$ is the radius of the shell, $\hat{P}_{R}$ is the corresponding conjugate momentum, $F_{\text {in,out }}=1-2 G m_{\text {in,out }} / R$ and $m_{\text {in,out }}$ are the Schwarzschild masses inside and outside the shell. This equation determines the trajectory of the shell $P_{R}(R)$ in the phase space of the model. Here the sign + or - is chosen in the right hand side of the equation depending on whether the shell is in-going or out-going.

For the bound motion we must use the symmetric $\left(P_{R} \rightarrow-P_{R}\right)$ form of constraint. 


$$
F_{\text {in }}+F_{\text {out }}-G^{2} M^{2} R^{-2}-\sqrt{F_{\text {in }} F_{\text {out }}} \operatorname{ch}\left(G P_{R} / R\right)=0
$$

The system which describes spherically symmetric gravitational field and a thin shell could be quantized in accordance with standard Dirac procedure. Then the classical constraint (3) is converted into a finite difference equation

$$
\begin{array}{r}
\Psi(m, \mu, S+i \zeta)+\Psi(m, \mu, S-i \zeta)= \\
\left(F_{\text {in }} F_{\text {out }}\right)^{-1 / 2}\left(F_{\text {in }}+F_{\text {out }}-M^{2} / 4 m^{2} S\right) \Psi(m, \mu, S)
\end{array}
$$

where $m=m_{\text {out }}, \mu=m_{\text {in }} / m_{\text {out }}$ and $S=R^{2} /(2 G m)^{2}=R^{2} / R_{g}^{2}$ is a dimensionless variable which measure the surface area of the shell. The finite shift parameter $\zeta=m_{p l}^{2} / 2 m_{\text {out }}^{2}$.

The quantum system described by the equation (田) possesses a discrete mass spectrum for the values of parameters $m_{\text {out }}<M$. The spectrum in the large black hole approximation is

$$
\left\{\begin{array}{l}
f_{1}(m, \mu, M)=\frac{(1+\mu)\left(2(1-\mu)^{2}-M^{2} / m^{2}\right)}{4 \zeta \sqrt{M^{2} / m^{2}-(1-\mu)^{2}}}=n \\
f_{2}(m, \mu, M)=\frac{M^{2} / m^{2}-(1-\mu)^{2}}{8 \zeta}-\frac{1}{2}=p
\end{array}\right.
$$

where $n$ is integer and $p$ is positive integer. If we take $m_{i n}=0$ which corresponds to the Carter-Penrose diagram of Fig. [ 1 we obtain the discrete mass spectrum for Schwarzschild mass $m=m_{\text {out }}$ parameterized by two quantum numbers $n$ and $p$ which was found in [2].

The first quantum number $n$ comes from ordinary boundary condition on the wave function at the infinity in the classically forbidden region . The origin of the second quantum number can be understood as follows. The classical motion of the shell may be of two types depending on the values of mass parameters, $m$ and $M$. If $M / m<2$ then we have the so called black hole motion. If $M / m>2$, then the shell forms the wormhole and it moves beyond the event horizon on the other side of the Einstein-Rosen bridge where we have another infinity. The configuration space in which $R$ takes values is a cross with two ends at the right and left space infinities of the Kruscal space-time, two ends in the future and past singularities $R=0$ and an intersection at the horizon $R=2 \mathrm{~m}$. But in quantum theory 
the wave function covers the regions on both sides of the Einstein-Rosen bridge for any values for mass parameters. So we have an additional independent boundary condition at the second infinity where the classical motion is also forbidden. It is the boundary condition at the second infinity that gives rise to the appearance of the second quantum number.

The quantum number which corresponds to the first quantization condition disappears for the unbound motion of the shell. But the second quantization condition remains valid and we could expect that there exist a quantum number for the unbound motion of the shell as well. Such qualitative considerations are supported by calculations in the large black holes [2] and quasiclassical [4] limits.

Analyzing the unbound motion we encounter the following difficulty. The solutions of equation (4) contain both in-going and out-going waves with equal amplitudes. This means that in classical limit we have a superposition of space-times with collapsing and expanding shells. But we'd like to have a single space-time in classical limit. This could be obtained by considering the quantum version of equation (1)

$$
\begin{aligned}
& \Psi(m, \mu, S+i \zeta)= \\
& \frac{1}{2 \sqrt{F_{\text {in }} F_{\text {out }}}}\left(F_{\text {in }}+F_{\text {out }}-\frac{M^{2}}{4 m^{2} S} \pm \frac{M}{m \sqrt{S}} \mathcal{Z}\right) \Psi(m, \mu, S)
\end{aligned}
$$

Analysis analogous to that carried out in [2] gives the following quantization condition

$$
6 f_{2}+f_{1}=2 \tilde{n}, \tilde{n}-\text { integer }
$$

where $f_{1}$ and $f_{2}$ are functions from (5). So the quantum number $\tilde{n}$ is a certain combination of quantum numbers for bound motion $n$ and $p$ (5). If we put $m_{i n}=0$ we obtain the spectrum for unbound motion which corresponds to the situation shown in Fig. 1]b. The spectrum for unbound motion depends on the continuous parameter $M$ instead of second discrete quantum number as it was explained at the beginning of the paper.

Now let us consider how radiation propagates in the field of black hole. We want to take into account the effect of back reaction of radiation on the gravitational.

We approximate the radiation by a thin null shell. The space-time near the light shell is divided into two regions. The inside region and the outside region are both characterized 
by their Schwarzschild masses $m_{\text {in }}$ and $m_{\text {out }}$. The wave packet takes away a small amount of the black hole mass $\delta m=m_{\text {out }}-m_{\text {in }}$.

The motion of the null shell is described by the constraint (10) with mass $M=0$ [5]. This means that

$$
\exp ( \pm G \hat{P} / R)=\sqrt{F_{\text {in }} / F_{\text {out }}}
$$

The sign "+" corresponds to the expanding motion of the shell and "-" to the collapsing motion.

When we go beyond the geometrical optics approximation, we need the corresponding wave equation, which gives trajectories (8) in geometric optics limit. This is just the equation (6) with $M=0$.

$$
\Psi(m, \mu, S-i \zeta)=\sqrt{F_{\text {in }} / F_{\text {out }}} \Psi(m, \mu, S)
$$

The global solutions of this equation exist only if the quantization condition

$$
3(1-\mu)^{2} / 8+\left(1-\mu^{2}\right) / 4=\zeta \tilde{n}, \tilde{n}-\text { integer }
$$

holds. It is just our Eqn. (7) for $M=0$. Now if the energy of the shell is small $\delta m \ll m_{\text {out }}$ , $m_{\text {in }} \approx m_{\text {out }} \approx m$, the Eqn. (10) takes the form

$$
\delta m \approx m_{p l}^{2} \tilde{n} / m, \tilde{n}-\text { integer }
$$

This is the radiation spectrum predicted by Bekenstein and Mukhanov [6].

The minimal energy of the light shell emitted by a black hole of Schwarzschild mass $m$ corresponds to $n=1$ :

$$
\delta m_{\min } \approx m_{p l}^{2} / m \sim T_{B H}
$$

It is of order of Hawking temperature $T_{B H}$ for the black hole.

So we have found that if the process of propagation of radiation emitted by a black hole is considered in a self-consistent way (the back reaction of radiation on gravitational field 
is taken into account), the spectrum of radiation turns out to be discrete and depends only on on the Schwarzschild mass of black hole (and not on its internal structure).

The mass energy spectrum of black hole in our model has a rather complicated structure ( (5) for bound motion and (7) for unbound motion). But the spectrum of radiation coming from black hole is not the spectrum determined by level spacings between mass-energy levels in the spectrum of black hole. It is because only quanta with energies satisfying the requirement (11) could propagate in the gravitational field of black hole in a self-consistent way.

Now we come to the problem whether the two spectra are in agreement. Let a quantum of energy $\delta m(12)$ is emitted by a black hole. Then the Schwarzschild mass has decreased: $m_{\text {out }}^{\prime}=m_{\text {out }}-\delta m$. So the parameters $n, p$ and $m_{\text {in }}$ must tune for the new $m_{\text {out }}$. This obviously could not be done only by changing $n \rightarrow n \pm 1, p \rightarrow p \pm 1$. We also have to change $m_{\in} \rightarrow m_{i n}^{\prime}$. So even if we had $m_{i n}=0$ before the emission we will have $m_{\text {in }} \neq 0$ after the emission. This could have a simple explanation. We have to conclude that not only a quantum is emitted to the infinity but necessarily another quantum must be emitted to the inside of the shell. It changes the mass $m_{i n}$ of the shell. So the quanta must be born in pairs. The situation is schematically presented on Fig. 2.

So there are several special features that the mechanism of Hawking radiation must possess which could be derived from our model.

1) Apart from requirement that radiation energy must correspond to the level differences in mass-energy spectrum (Bohr postulate) we have to consider also the requirement that radiation is able to propagate in the gravitational field of the black hole. It is this last requirement that leads to the fact that a black hole of mass $m$ emits radiation with energies of order of Hawking temperature.

2) Quanta of radiation are emitted in pairs. Together with quantum emitted to the infinity there is always a quantum which falls into the black hole. We traced out this second quantum in our model by noting that the emission of radiation leads to the change of Schwarzschild mass inside the shell. In usual quantum mechanics the process of emission 
of radiation causes the transition from one energy level to another in a given quantummechanical system. Here we see that the emission leads to the change in the inner structure of black hole and changes the mass spectrum itself.

There is yet another interesting feature of the Hawking radiation in our model. Let us consider the situation when the mass of black hole is small, that is at the end of the evaporation process. Then we need the exact formula (10)

$$
(\delta m)^{2} / 8+m_{\text {out }} \delta m / 2=\tilde{n} m_{\text {pl }}^{2} / 2, \tilde{n}-\text { integer }
$$

This is a quadratic equation for $\delta m$. It's positive solution is

$$
\delta m=-2 m_{\text {out }}+2 \sqrt{m_{\text {out }}^{2}+\tilde{n} m_{\text {pl }}^{2}}
$$

The minimal energy of quanta emitted corresponds to $n=1$. But if

$$
m_{\text {out }}<-2 m_{\text {out }}+2 \sqrt{m_{\text {out }}^{2}+m_{\text {pl }}^{2}}
$$

the energy of the emitted quantum would be greater then the mass of the black hole. So the last feature is

3) Black holes with masses

$$
m_{\text {out }}<\frac{2}{\sqrt{5}} m_{p l}
$$

do not radiate. The evaporation of black holes leads in our model to remnants of about Planckian mass.

The main qualitative result of the present letter is the following. We found that due to the nontrivial structure of configuration space (which reflects the complex structure of the complete Schwarzschild manifold) the selfgravitating null shells have discrete energy spectrum. We consider such null shell as describing approximately the quantum Hawking radiation. It appeared that the spectrum of the radiation does not math the spectrum of the massive selfgravitating thin shell. The way out of this difficulty is to suppose that collapsing shell radiates not only to infinity but also inward. the ingoing radiation leads to the change 
of the mass $m_{\text {in }}$ inside the shell (even it is initially zero). Thus the inner structure of the black hole is changing during its evaporation. The distant observer however will see only the radiation spectrum which is that predicted by J.Bekenstein and V.Mukhanov for black hole masses much larger than the Planckian mass. As a byproduct we show that the black holes with the masses less than the Planckian mass do not radiate. 


\section{REFERENCES}

[1] K.Kuchar, Phys. Rev. D 50, 3961, (1994)

[2] V.A.Berezin, A.M.Boyarsky, A.Yu.Neronov, Phys. Rev. D 57, 1118, (1998)

[3] L.D.Landau, E.M.Lifshitz, Quantum mechanics, Nauka, Moscow, (1974)

[4] A.Yu.Neronov, Quasiclassical mass spectrum of quantum black hole model with selfgravitating dust shell. gr-qc/9808021

[5] J.Louko, B.F.Whitting, J.L.Friedman, Phys. Rev. D 57, 2279, (1998)

[6] J.Bekenstein, A.Mukhanov, Phys.Lett. B 360, 7 (1995) 


\section{FIGURES}

FIG. 1. Space-time with a collapsing shell: bound (a) and unbound (b) trajectories of the shell.

FIG. 2. Process of emission of quanta of radiation. 


$$
\hat{\Delta}^{\circ}
$$




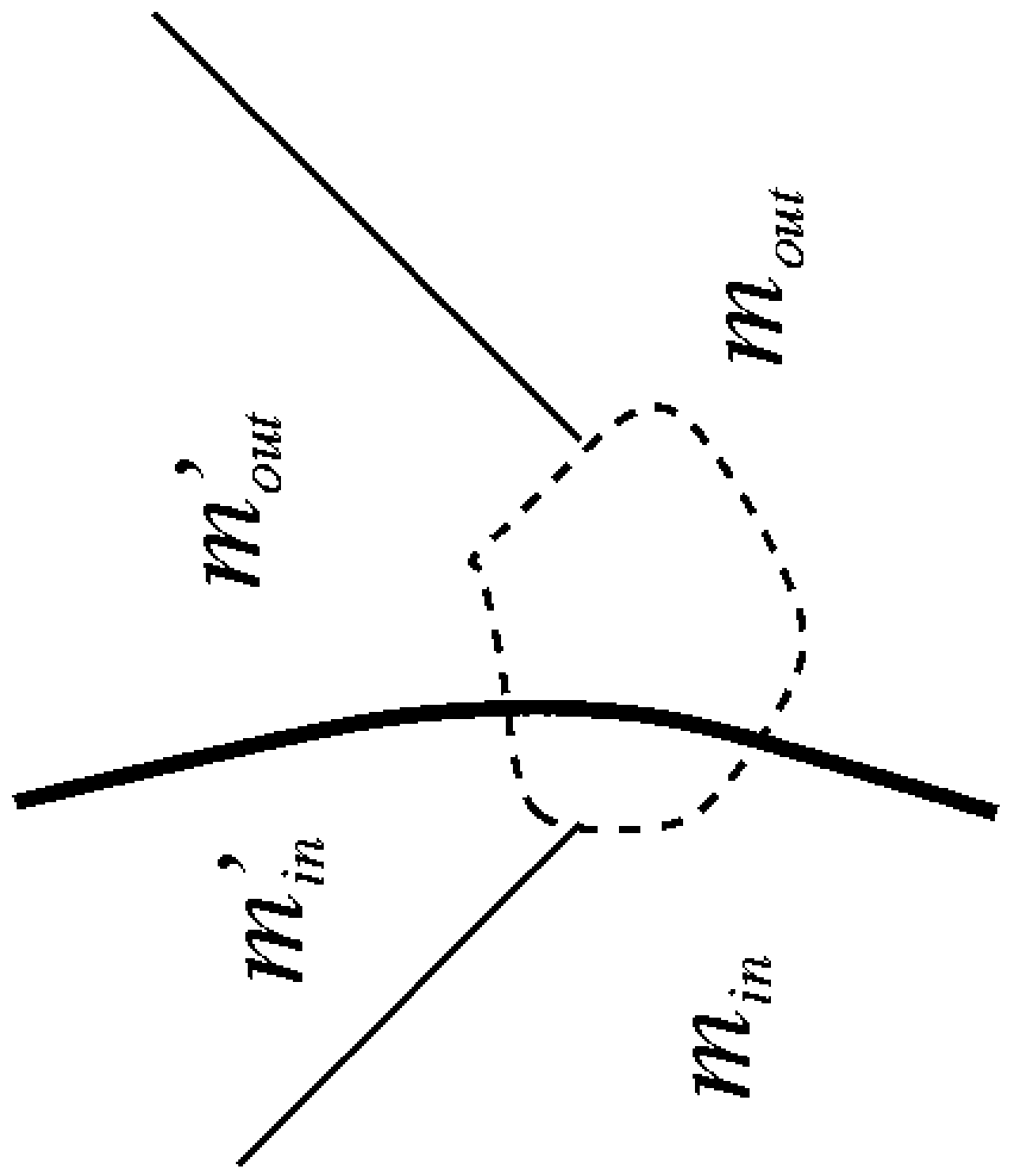

